\title{
Evaluation of Risk Factors and Outcome of Haemorrhagic Stroke in Neonates
}

\author{
AbdelrahimAbdrabou Sadek ${ }^{1}$, Ramadan Abo El Hassan A. ${ }^{2}$, Nahla M. Gamal ${ }^{3}$. \\ (1)Assistant Professor of neuropediatrics, Sohag faculty of medicine. (2)Lecturer of Pediatrics, Sohag faculty \\ of medicine. (3)Resident of Pediatrics, Sohag faculty of medicine.
}

\begin{abstract}
Back ground: Few data regarding causes and outcomes of hemorrhagic stroke in late preterm and term neonates are available so we were trying to identify them in this study.

Methodes:This prospective case control study was carried out at Sohag University Hospitals, during the period from 1/1/2015 to 31/12/2015 at the department of pediatrics and neonatal care unit at Sohag University. A total of $\mathbf{5 0}$ neonates term and late preterm neonates ( $\mathbf{3 4}$ weeks gestation) and $\leq \mathbf{2 8}$ days of life had met the inclusion criteria were $\mathbf{2 3}$ males and $\mathbf{2 7}$ females.

Results: As regard outcome in our study, $8 \%$ had grade I, $14 \%$ had grade II, 32\% had grade III, $12 \%$ had grade IV, $\mathbf{1 4 . 5 \%}$ had subdural hemorrhage and $\mathbf{2 0 \%}$ had subarachnoid hemorrhage. So most patients have grade III intracranial hemorrhage and subarachnoid hemorrhage. According to the severity, in our study patients who had mild intracranial hemorrhage represented $\mathbf{4 8 \%}$ and patients with moderate intracranial hemorrhage represented $\mathbf{3 0 \%}$ and patients with severe intracranial hemorrhage represented $\mathbf{2 2 \%}$.

Conclusion: IVH is very rarely reported in full-term and late preterms neonates and may occur in these children with a variety of risk factors, in our study we tried to identify these risk factors and also the outcome and we found that the most frequent associated risk factors were absence of vitamin $\mathrm{K}$ administration followed by prolonged labour then neonatal sepsis.
\end{abstract}

\section{Introduction}

Hemorrhagic stroke (HS) defined as intraventricular, intraparenchymalintraventricularhemorrhage is common and usually caused by germinal matrix hemorrhage. While it is less common in late preterm (34-37) wks and term neonates.Much less is known about risk factors and outcome of HS in late preterm (34 -37) weeks and term neonates (1).

Incidence of symptomatic HS in term neonates has recently been estimated at $\mathbf{0 . 1 7 / 1 0 0 0}$ live births (2). Hemorrhage location often dictates the clinical course. Symptoms can include seizures or may be non-specific like apnea, respiratory distress, fever or poor feeding (3).

Brouewer AJ have reported impairment in (9$\mathbf{5 7 \%}$ ) of these children though the higher estimate comes from a study that included neonates with isolated subdural hemorrhage. Most studies report mortality rates from $1 \%$ to $25 \%$ (4).Intracranial hemorrhage in term newborn has been increasingly recognized but the occurrence and risk factors in late preterm are still unclear (5).
Several risk factors have been reported in term newborns with ICH. Maternal risk factors causing ICH in the first neonatal week includes usage of drugs (such as aspirin, cocaine), pregnancy-induced hypertension, placental abruption, placental alloimmunization and autoimmune disorders. Major perinatal risk factor are birth trauma, low Apgar score, resuscitation at birth, thrombocytopenia, breastfed infants who received no vitamin $\mathrm{K}$, inherited coagulopathy, disseminated intravascular coagulopathy, increased cerebral venous pressure, prolonged labor, unassisted vaginal delivery, forceps delivery and suction cup(6).

Aim of the work: The work aimed to identify the risk factors and outcome of hemorrhagic stroke in neonates $\geq \mathbf{3 4}$ weeks of gestational age and $\leq \mathbf{2 8}$ days of life in Sohage University Hospital.

Patients \& Methods:

Type of the Study: prospective hospital based study.

Place of the study: Neonatal intensive care unit (N.I.C.U) and Neuropediatric Outpatient Clinic at Sohage University Hospital. 
Study Period: one year (from 1-1-2015 to 3112-2015)

Patients:

Inclusion Criteria: About 50 term and late preterm $(\geq \mathbf{3 4}$ weeks gestation) neonates who diagnosed as having definite hemorrhagic stroke by neuroradiological studies (transcranial U/S,CT,MRI) presented to N.I.C.U during the period of the study were included.

\section{Exclusion Criteria: -}

1-The prescence of major congenital malformation of the nervous system,e.ghydraencephaly ,anencephaly , encephalocele and hydrocephalus.

2-Early preterm neonates $(\leq \mathbf{3 4}$ weeks gestation).

Ethical consideration: Approval of Sohag Faculty of Medicine Research Ethics Committee was obtained. Written consent was obtained from guardians.

Methods of the study

(A) Detailed clinical data of the patients were retrospectively collected with special reference to
1- The antenatal, perinatal and postnatal events as regard recording (Apgar score, Thompson score and Perinatal score index) and focusing on administration of vitamin $\mathrm{K}$.

2-The presenting symptoms focusing on nervous system as convulsions, abnormal movements, conscious level and motor symptoms.

3- Family history of inherited bleeding tendency.

(B) Data of patients examination focusing on neurological system as conscious level, abnormal features, other congenital anomalies, motor system and cranial nerves examination.

(C) Investigations: complete blood count (CBC) and complete coagulation profile (prothrombinetime,prothrombine concentration and activated partial thrombplastin time).

(D) Brain imaging (CT, MRI, transcranial U/S).

(E) Follow up of the patients after 6 months at Neuropediatric Outpatient Clinic by developmental assessment \& neurological imaging (CT, MRI).

\section{Results}

A total of $\mathbf{5 0}$ neonatesterm and late preterm neonates ( $\geq \mathbf{3 4}$ weeks gestation) and $\leq \mathbf{2 8}$ days of life had met the inclusion criteria were $\mathbf{2 3}$ males and $\mathbf{2 7}$ females with the mean age $\mathbf{1 3 . 5 6}$ and range from 2$\mathbf{2 8}$ days. 50\% of the deliveries were normal vaginal delivery and $\mathbf{5 0 \%}$ were cesarean section with $\mathbf{2 8 \%}$ have difficult labour and $\mathbf{7 2 \%}$ have no difficult labour .6\% experienced instrumental deliveries. Neonates were deliveries with Apgar score mean 8.8 ranges from 7 to 10. Pregnancy induced hypertension was in $4 \%$ of cases. $6 \%$ of cases showed fetal distress .Vitamin $\mathrm{K}$ was administered in 64\%, $6 \%$ had not administered their dose and 3\% not known. 8\% had evidence of neonatal sepsis, $6 \%$ had no evidence of neonatal sepsis and $\mathbf{4 3 \%}$ not known. As regard outcome, in our study $8 \%$ had grade I, $14 \%$ had grade II, 32\% had grade III, 12\% had grade IV, 14\% had subdural hemorrhage and $\mathbf{2 0 \%}$ had subarachnoid hemorrhage. So most patients had grade III intracranial hemorrhage (32\%) followed subarachnoid hemorrhage $\mathbf{( 2 0 \% )}$ ) followed by grade II intracranial hemorrhage (14\%).

\section{Discussion}

The present study performed a clinical analysis and reviewing the data of $\mathbf{5 0}$ term and late preterm neonates ( $\geq \mathbf{3 4}$ weeks gestation) and $\leq \mathbf{2 8}$ days having intracranial hemorrhage by neuroradiological findings (CT or MRI Brain or transcranial U/S) with special consideration for the risk factors and neurological out come. The study included a total of $\mathbf{5 0}$ neonates having intracranial hemorrhage with age range between $\mathbf{3 4}$ wks of gestational age to less than $\mathbf{2 8}$ days of life. The median age was $\mathbf{1 2}$ days and there were 23 males (46\%) and 27 females (54\%). The male to female ratio was $(\mathbf{1 : 1 . 2})$. This ratio was close to the study ofBruno, et al., 2014 where male to female ratio was (1:1). The mean age incidence was $\mathbf{1 3 . 5 6}$ days which was higher than that of study of Bruno, which was 7dayes (7).

Concerning the median gestational age of the studied population is $37 \mathrm{wk}$ range from $34 \mathrm{wks}$ to 39wks ,which is less that of Burno et al., that show median gestational age 39.7wks, range from 38 wks to $\mathbf{4 0 . 7 w k s ~ ( 7 ) ~ a n d ~ i t ~ w a s ~ a l s o ~ l e s s ~}$ than that of Afsharkhaset al., 2015 $(38$ wks)(8). 
Intracranial hemorrhage in late preterm and full term neonates is multifactorial, in our study cases had two or more risk factors but the most associated risk factors were prolonged labor $\mathbf{( 2 8 \% )}$ and patients who did not administered vitamin $\mathrm{K} \mathbf{( 3 0 \% )}$. As regard risk factors of intracranial hemorrhage, mode of delivery is one of the most important factors that had been implicated as a cause of intracranial hemorrhage. In our study $\mathbf{5 0 \%}$ had been delivered by normal vaginal delivery and the other $\mathbf{5 0 \%}$ has been delivered by cesarean section which was different from the result of Bruno et al., in which $\mathbf{7 2 \%}$ had been delivered by normal vaginal delivery and $\mathbf{2 8 \%}$ by cesarean section (7). Instrumental use during delivery was used in $\mathbf{3 \%}$ of our patients.

Towner et al., reviewed that the rate of intracranial hemorrhage was higher among infants delivered by vacuum extraction, forceps, or cesarean section than among infants delivered vaginally without instrumentation. The increased risk of intracranial hemorrhage among infants delivered by vacuum extraction or forceps, however, may be the result of common risk factors during abnormal labor (9).

In our study, Apgar score mean at $\mathbf{5}$ minutes was $\mathbf{8 . 8}$ in contrast to the study ofJahwar et al., the mean Apgar scores at $\mathbf{5}$ minutes for case patients was 6.0.AlsoJahwar et al.,reported that among 11 cases of term neonates, intracranial hemorrhage was evidenced in all $\mathbf{3}$ of the infants who had 5-minute Apgar score of $\mathbf{7}$ or less also reported that low Apgar scores, with and without requirement for resuscitation at birth, are risk factors for intracranial hemorrhage (10). A significant clinical problem, which is generally regarded as one of the risk factors of $\mathrm{IVH}$, is asphyxia, a secondary consequence to an interruption of placental blood flow and decreased blood flow to the brain and impairment of cerebral autoregulation. Treatment with therapeutic hypothermia in neonates with hypoxic ischemic encephalopathy may prevent the development of severe brain injuries and improve the long- term outcome. On the other hand, it places newborns at greater risk of IVH by causing fluctuations of the cerebral blood flow, depressed cardiac function, hypotension, and changes in coagulation cascade (11).
In our study, foetal distress is recorded in $6 \%$ of our cases which is lower than the study of Bruno et al., in which patients with respiratory distress reported in $\mathbf{2 1 \%}$ of cases (7).

Patients who did not administered vitamin $\mathrm{K}$ was $30 \%$ of cases this result was close to Hubbardet al., that reported that vitamin $\mathrm{K}$ is essential for the final carboxylation of coagulation factors II, VII, IX, and X (12 ). Vitamin $\mathrm{K}$ deficiency can lead to hemorrhagic disease of the newborn, which is rare in the United States because of routine administration of vitamin $\mathrm{K}$ but thereare reports of intracranial hemorrhage with this deficiencyIt is most common in exclusively breast-fed infants who received no vitamin $K$ after birth, and also in infants with mothers taking various antiepileptic drugs, analgesics or street drugs (13).

Neonatal sepsis had been reported in $6 \%$ of cases of our study, sepsis can lead to intracranial hemorrhage by several ways such as thrombocytopenia and disseminated intravascular coaguolopathy. Disseminated intravascular coagulation can also lead to severe thrombocytopenia and intracranial hemorrhage in the term newborn, but this occurs in the sick newborn, usually in the neonatal intensive care unit setting (14).

In our study, the most frequent presentation was convulsions $(\mathbf{5 0 \%})$ followed by pallor $\mathbf{( 2 0 \% )}$ and poor suckling $\mathbf{( 2 2 \% )}$ ). Indeed, intracranial hemorrhage is a common cause of neonatal seizures. The seizure occurrence, type, and severity are variable in term newborns. Seizure may occur soon after birth or after an extended period of normality days after birth. Neonatal seizures may prove difficult to differentiate from normal movement, or may have variable presentations, ranging from a subtle seizure such as apnea to a more apparent seizure to status epilepticus (15).

Apnea was one the most important clinical manifestations it represented $\mathbf{1 6 \%}$. Apnea of any duration associated with significant tachycardia or bradycardia in a newborn who is otherwise apparently well should prompt the physician to consider intracranial abnormalities such as intracranial hemorrhage Temporal lobe hemorrhage with apneic seizures in term neonates although uncommon, has been reported (16). 
On the other hand, apnea with bradycardia may be a manifestation of elevated intracranial pressure (17).

Concerning hematological investigations, $54 \%$ of patients had prolonged PT and aPTT and $24 \%$ had thrombocytopenia which was near the study of

Jahwar et al., and also near to the study of

(Afsharkhas et al, 2015)

in which $50 \%$ of neonates had coagulation profile disorders including DIC (20\%), sinovenous thrombosis (16.7\%) and thrombocytopenia $(\mathbf{1 3 . 3 \%})$ of cases $(\mathbf{8 , 1 0})$.

Sandberg et al.,Identified $\mathbf{1 1}$ term newborns with spontaneous intraparenchymal hemorrhage; coagulopathy was noted in $\mathbf{3}$ of the 11. Thrombocytopenia is the most common condition leading to intracranial hemorrhage in term newborns. Thrombocytopenia has multiple causes; it may be drug induced, infectious, genetic, or immune-related, or due to disseminated intravascular coagulation or placental insufficiency . The risk for intracranial hemorrhage increases with severity of thrombocytopenia. Most cases occur with

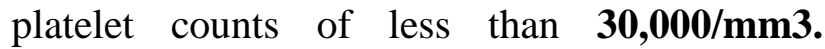
Neonatal alloimmune thrombocytopenia is a particularly important risk for intracranial hemorrhage: $\mathbf{7 - 2 6 \%}$ of affected neonates develop intracranial hemorrhage (15).

Intracranial haemorrahge can be classified according to the degree as follow: grade I the mildest form of IVH that bleeding was limited to the lining of the ventricles), grade II (the blood does spilled into the ventricles, but there was no enlargement or swelling), grade III (the ventricles had become enlarged and are full of blood), and grade IV (Blood spilled out from the ventricles into the surrounding brain).In our study $\mathbf{8 \%}$ had grade I, $14 \%$ had grade II, 32\% had grade III, $\mathbf{1 2 \%}$ had grade IV, $\mathbf{1 4 . 5 \%}$ had subdural hemorrhage and $\mathbf{2 0 \%}$ had subarachnoid hemorrhage. So most patients had grade III intracranial hemorrhage followed by subarachnoid hemorrhage $\mathbf{2 0 \%}$, this was different from the study of (Afsharkhas et al., 2015) in which most patients had grade $1(33 \%)$ followed by grade II (20\%) followed by grade IV (15\%) (8).

Also intracranial hemorrhage can be classified according to the severity into mild intracranial hemorrhage which involves only one compartment or one lobe, with a maximum midline shift of $0.5 \mathrm{~cm}$, or as intraventricular hemorrhage in only one ventricle with no hydrocephalus. Moderate intracranial hemorrhage is defined as hemorrhage involving only one lobe and compartment with midline shift or as intraventricular hemorrhage of more than one ventricle but no hydrocephalus; when two or more lobes are involved, midline shift is inconsequential. Severe intracranial hemorrhage is defined as hemorrhage in more than one lobe and in more than one compartment or as intraventricular hemorrhage with hydrocephalus. In our study grade I is considered mild, grade II moderate, and grade III \& IV severe. In our study mild intracranial hemorrhage represent $\mathbf{4 8 \%}$ and patients with moderate intracranial hemorrhage represents $30 \%$ and patients with severe intracranial hemorrhage represents $22 \%$ so most patients in our study had mild intracranial hemorrhage and the least had severe intracranial hemorrhage this is close to the study of Afsharkhas et al., in which most patients had mild intracranial hemorrhage $\mathbf{( 3 3 \% )}$ followed by moderate intracranial hemorrhage which represented $\mathbf{( 3 0 \% )}$ followed by severe intracranial hemorrhage which represented (22\%) (8).

As regard the outcome; there is no single predictor of intracranial hemorrhage outcome in newborns. However, the extent (compartmental, lobar, or both), the severity, and the etiology of intracranial hemorrhage may influence the prognosis. In addition, low gestational age (i.e., prematurity), early occurrence of recurrent seizures or status epilepticus, and the need for multiple anticonvulsants to control seizures were associated with poor outcome (18).Afsharkhas et al.,also reported thatthe consequences of IVH are dependent to the grade of bleeding. So that, mortality and sequelae due to $\mathrm{IVH}$, ranged from $\mathbf{5 \%}$ in grade I to $\mathbf{9 0 \%}$ in grade IV, respectively (8). The majority of newborns with spontaneous subarachnoid hemorrhage will make a complete recovery. Disability is more likely to occur in a newborn with frontal lobe hemorrhage or when multiple intracranial compartments are involved and this was confirmed with our study that report that all patients with subarachnoid hemorrhage had no 
neurological abnormalities in the follow up and patients who had grade III intracranial hemorrhage associated with the highest incidence of neurological abnormalities $(\mathbf{7 1 . 4 3 \% )}$.

33\% of patients had abnormal neurological outcome in the follow up at the age of six months in the form of hypotonia, spasticity, hydrocephalus , apathy and poor activity.The incidence of neurological abnormality in our study was lower than that of ofBruno et al., in which nearly half of survivors demonstrated a neurologic deficit at the last follow-up, and approximately $56 \%$ of subjects required physical, occupational, and/or speech therapy services (7). Hydrocephalus in our study had been reported in $\mathbf{6}$ cases that required surgical intervention.

\section{Summary and conclusion:}

This study carried out at Neonatal Care Unit and Pediatric Neurology Clinic at Sohag University Hospitals, during the period from $1 / 1 / 2015$ to 31/12/2015. The study included a total of $\mathbf{5 0}$ neonates meeting the inclusion criteria having intracranial hemorrhage with age range $(\geq \mathbf{3 4}$ weeks gestation and $\leq \mathbf{2 8}$ days of life). The median age was $\mathbf{1 2}$ days and there were $\mathbf{2 3}$ males (46\%) and 27 females (54\%). The male to female ratio was (1:1.2).

As regard outcome in our study, $\mathbf{8 \%}$ had grade I, $14 \%$ had grade II, 32\% had grade III, 12\% had grade IV, $\mathbf{1 4 . 5 \%}$ had subdural hemorrhage and $\mathbf{2 0 \%}$ had subarachnoid hemorrhage. So most patients have grade III intracranial hemorrhage and subarachnoid hemorrhage.All newborns with subarachnoid hemorrhage showed a complete recovery. Disability is more likely to occur in a newborn with frontal lobe hemorrhage or when multiple intracranial compartments are involved and this was confirmed in our study which showed that all patients with subarachnoid hemorrhage had no neurological abnormalities in the follow up and patients who had grade III intracranial hemorrhage associated with the highest incidence of associated neurological abnormalities $\mathbf{( 7 1 . 4 3 \% ) . ~ 3 3 \% ~ o f ~ p a t i e n t s ~ h a d ~}$ abnormal neurological outcome in the follow up at the age of six months in the form hypotonia, spasticity, hydrocephalus, apathy and poor activity. These patientsrequired physical, occupational, and/or speech therapy services.
Hydrocephalus has reported in $\mathbf{6}$ cases in our study that required surgical intervention.

\section{Refferences}

1- Saver JL, Warach S, Janis S, et al., (2005): Standardizing the structure of stroke clinical andScheel JR, Ray J, Gage FH, et al. Quantitative analysis of gene expression in living adult neural stem cells by gene trapping. Nat Methods.2:363.

2- Takenouchi T, Kasdorf E, Engel M, et al., (2012): Changing pattern of perinatal brain injury in term infants in recent years. PediatrNeurol; 46:106-10.

3- Brouwer AJ, Groenendaal F, Koopman C, et al., (2010): Intracranial hemorrhage in fulltermnewborns: a hospital-based cohort study. Neuroradiology 52:567-76.

4- Ou-Yang MC, Huang $\mathrm{CB}$, Huang $\mathrm{HC}$, et al., (2010): Clinical manifestations of symptomaticintracranial hemorrhage in term neonates: 18 years of experience in a medicalcenter. PediatrNeonatol; 51:208-13.

5- Sirgiovanni I, Avignone S, Groppo M, Bassi L, Passera S, Schiavolin P, Lista G, Cinnante C, Triulzi F, Fumagalli M, Mosca F (2014):PediatrRadiol. 44(3):289-96. doi: $10.1007 / \mathrm{s} 00247$.

6- Blankenberg FG, Loh NN, Bracci P, et al., (2000): Sonography, CT, and MR imaging: a prospective comparison of neonates with suspected intracranial ischemia and hemorrhage. AJNR 21(1):213-218.

7- Bruno CJ, Beslow LA, Witmer CM, Vossough A, Jordan LC, Zelonis S, Licht DJ, Ichord RN, Smith SE (2014): Hemorrhagic stroke in term and late preterm neonates. Arch Dis Child Fetal Neonatal Ed 99(1):48-53.

8- Afsharkhas L, Khalessi N, KarimiPanah M (2015):Intraventricular hemorrhage in term neonates: sources, severity and outcome. Iran J Child Neurol 9(3):34-39.

9- Towner D, Castro MA, Eby-Wilkens E, Gilbert WM (1999): Effect of mode of delivery in nulliparous women on neonatal intracranial injury. $\mathrm{N}$ Engl J Med. 2. 341(23):1709-14. 
10- Jhawar BS, Ranger A, Steven DA, Del Maestro RF (2005): A follow-up study of infants with intracranial hemorrhage at fullterm. Can J Neurol Sci;32:332-9.

11-Yazidi GA, Srour M, Wintermark $P$ (2014): Risk factors for intraventricularhemorrhage in term asphyxiated newborns treated with hypothermia. PediatrNeurol 50:630-63.

12-Hubbard D, Tobias JD (2006): Intracerebral hemorrhage due to hemorrhagic disease of the newborn and failure to administer vitamin $\mathrm{K}$ at birth. South Med J;99:1216-20.

13-Karlowicz MG, White LE(1993): Severe intracranial hemorrhage in a term neonate associated with maternal acetylsalicylic acid ingestion. ClinPediatr (Phila);32:7403.
14-Gupta SN, Kechli AM, Kanamalla US (2009): Intracranial hemorrhage in term newborns: management and outcomes. Pediatr Neurol. 40(1):1-12.

15- Sandberg DI, Lamberti-Pasculli M, Drake JM, Humphreys RP, Rutka JT (2001): Spontaneous intraparenchymal hemorrhage in full-term neonates. Neurosurgery; 48:1042-8.

16- Sirsi D, Nadiminti L, Packard MA, Engel M, Solomon GE (2007): Apneic seizures: a sign of temporal lobe hemorrhage in full-term neonates. Pediatr Neurol;37:366-70.

17- Fenichel GM (2007): Neonatal hypoxicischemic and hemorrhagic cerebral injury. In: Neonatal neurology. 4th ed. Philadelphia: Churchill Livingstone Elsevier, 69-87.

18- Pisani F, Cerminara C, Fusco C, Sisti L (2007): Neonatal status epilepticusvs recurrent neonatal seizures: clinical findings and outcome. Neurology; 69:2177-85. 
SOHAG MEDICAL JOURNAL

Vol. 21 No.1 Jan 2017
Evaluation of Risk Factors and Outcome of Haemorrhagic Stroke in Neonates Nahla M. Gamal 